\section{Chelsea Hospital for Women.}

"I consider the Course was very well worth while. I consider the lectures and teaching were excellent, and the Staff without exception were most courteous and helpful. Sometimes one wished that there were more opportunity for asking questions arising out of the lectures, though I realize this might waste time for other members of the class. Some details as to preparation of patients for operation and post-operative treatment would have been interesting to me personally, as in out of the way places one cannot leave it to the Nursing Staff."

"I have found this a very helpful Course. Perhaps it could with advantage have been slightly shortened, as some of the subjects slightly overlapped. I would like to have been taken round the wards and had the post-operative treatment and medical gynæcological treatment demonstrated. Could the subject of Birth Control be included in this Course? Some teaching on these lines is greatly needed."

\section{London Clinic and Institute of Physical Medicine.}

"I would suggest practical demonstrations, especially with regard to diathermy with regard to : application of electrodes, types of electrodes, precautions and average doses, clinical uses (and abuses), uterine and rectal diathermy. For my part I would much prefer that all subjects should be treated from an elementary viewpoint which would be of use to the $G$. P. rather than of interest to the specialist."

"I was unable to attend the Saturday morning lectures. The rest of the Course I thought good. The actual demonstration of methods being used is what is wanted and there was general favourable comment that it was used here."

"A moderate Course and somewhat uneven. The gynæcological lecture was good. A lecture from the diathermy ma年 would have been welcome. The lectureng on Rheumatism and on Nervous Diseases were too enthusiastic and failed to give balanced view. Their cases were obvious selected."

"I thought this was a very valuab居 Course with much information given in $\frac{9}{9}$ short space of time. I should suggest that the two lectures should follow each othep at once, or with only (say) ten minutes interval."

"Very good. Might I suggest that it not very easy for some of us from the outskirts of town to reach Victoria bs 9.30 a.m. If a quarter of an hour could be taken off the mid-morning break and the first lecture start a quarter of an hour later it might prevent the first lecture having to put up with a stream of lat comers."

\section{REVIEW.}

Diseases of The Skin. By the late Robefe W. MacKenna, M.A., M.D. Thir Edition, revised and'enlarged, by R. M. ß MacKenna, M.A., M.D., M.R.C.P. coloured plates and 149 illustration?. Baillière, Tindall and Cox. Price 25s.을

This book gives a very practical and com: prehensive account of all the common ski diseases.

It is well arranged, the diseases being grouped according to their ætiology, which is in itself a help to the student. It is easy and interesting to read, containing very litte padding, but nevertheless it deals thoe oughly with all essential details and, unlike a number of textbooks, it gives practical details regarding treatment. The illustras tions, consisting of photographs and two colo oured plates, are excellent and this feature alone raises the book above its fellows. If should prove a boon to students and gener practitioners, and the dermatologist will fine it handy for a quick reference.

J. R. S. S. $\frac{0}{\stackrel{0}{D}}$ 